\title{
Nonunitary Lagrangians and Unitary Non-Lagrangian Conformal Field Theories
}

\author{
Matthew Buican and Zoltan Laczko \\ CRST and School of Physics and Astronomy Queen Mary University of London, London E1 4NS, United Kingdom
}

(Received 7 December 2017; published 23 February 2018)

\begin{abstract}
In various dimensions, we can sometimes compute observables of interacting conformal field theories (CFTs) that are connected to free theories via the renormalization group (RG) flow by computing protected quantities in the free theories. On the other hand, in two dimensions, it is often possible to algebraically construct observables of interacting CFTs using free fields without the need to explicitly construct an underlying RG flow. In this Letter, we begin to extend this idea to higher dimensions by showing that one can compute certain observables of an infinite set of unitary strongly interacting four-dimensional $\mathcal{N}=2$ superconformal field theories (SCFTs) by performing simple calculations involving sets of nonunitary free four-dimensional hypermultiplets. These free fields are distant cousins of the Majorana fermion underlying the two-dimensional Ising model and are not obviously connected to our interacting theories via an RG flow. Rather surprisingly, this construction gives us Lagrangians for particular observables in certain subsectors of many "non-Lagrangian" SCFTs by sacrificing unitarity while preserving the full $\mathcal{N}=2$ superconformal algebra. As a by-product, we find relations between characters in unitary and nonunitary affine Kac-Moody algebras. We conclude by commenting on possible generalizations of our construction.
\end{abstract}

DOI: $10.1103 /$ PhysRevLett.120.081601

Introduction.-Free fields in two spacetime dimensions are versatile: operators, correlation functions, and partition functions of interacting conformal field theories (CFTs) can often be constructed algebraically from free bosons via the Coulomb gas formalism, and the simplest unitary minimal model - the Ising model— has a free Majorana fermion underlying it (see [1] for a review). Free fields in higher dimensions seem less powerful: in order to have something useful to say about an interacting CFT, one must usually labor to connect such free fields to the CFT in question through a suitably "smooth" path in the space of couplings [2].

However, one may hope to overcome these obstacles in $d>2$ spacetime dimensions whenever there are relations between quantum field theories (QFTs) in $d$ dimensions and QFTs in 2D. In the case of 4D superconformal field theories (SCFTs) with at least $\mathcal{N}=2$ supersymmetry (SUSY), one such relation was given in [3]: the sector of so-called "Schur" operators of the 4D SCFT (briefly reviewed in the Supplemental Material [4]) is isomorphic to a $2 \mathrm{D}$ chiral algebra living on a plane, $\mathcal{P} \subset \mathbb{R}^{4}$. On the chiral algebra side of this relation, one of the most basic quantities we can compute is the torus partition function

Published by the American Physical Society under the terms of the Creative Commons Attribution 4.0 International license. Further distribution of this work must maintain attribution to the author(s) and the published article's title, journal citation, and DOI. Funded by SCOAP ${ }^{3}$.

$$
Z(x, q) \equiv q^{-\left(c_{2 d} / 24\right)} \operatorname{Tr} x^{M^{\perp}} q^{L_{0}},
$$

where the trace is over the Hilbert space of states associated with the chiral algebra, $c_{2 d}$ is the chiral algebra central charge, $M^{\perp}=j_{1}-j_{2}$ is the spin transverse to $\mathcal{P}\left[j_{1,2}\right.$ are Cartans of $S O(4)], q \in \mathbb{C}$ is a fugacity, and $L_{0}$ gives the holomorphic scaling dimension, $h$. On the 4D side of the relation, (1) is mapped to a particular refined Witten index, called the Schur index [5] (see the Supplemental Material [4] for further details), that counts the Schur operators weighted by certain quantum numbers

$$
\mathcal{I}_{\mathrm{S}}(q) \equiv q^{c_{4 d} / 2} \operatorname{Tr}_{\mathcal{H}}(-1)^{F} q^{E-R}=Z(-1, q),
$$

where $c_{4 d}$ is the $4 \mathrm{D} c$ central charge, $F$ is fermion number, $E$ is the scaling dimension, and $R$ is the $s u(2)_{R}$ weight (clearly, the holomorphic scaling dimension satisfies $h=$ $E-R$ while $c_{2 d}=-12 c_{4 d}$ [3]). Note that both (1) and (2) can be refined by additional flavor fugacities (i.e., fugacities for symmetries that commute with $\mathcal{N}=2$ SUSY in 4D), but such modifications will not play a role in our discussion below.

While we believe that many of the ideas we will present are quite broadly applicable (with suitable modifications), in this Letter, we specialize to a particular infinite set of strongly coupled SCFTs whose simplest member is the socalled $\left(A_{1}, D_{4}\right)$ theory [6]. In this class, the manipulations we use are particularly simple.

The Schur index for the $\left(A_{1}, D_{4}\right)$ theory was computed in [9-12] and was shown to equal the vacuum character of 
$\widehat{s u(3)_{-\frac{3}{2}}}$ (as conjectured in [13]). More recently, the authors of [14] proposed that this unflavored Schur index takes the following simple form:

$I_{S}^{\left(A_{1}, D_{4}\right)}(q)=q^{\frac{1}{3}}$ P.E. $\left(8 \frac{q}{1-q^{2}}\right) \equiv q^{\frac{1}{3}} \exp \left(8 \sum_{n=1}^{\infty} \frac{1}{n} \frac{q^{n}}{1-q^{2 n}}\right)$,

and this formula was proven in [15] (see, also, the discussion in [16]) to be equivalent to the vacuum character of $\widehat{s u(3)})_{-\frac{3}{2}}$ [17]. Interestingly, under the rescaling $q \rightarrow q^{\frac{1}{2}}$,

(3) reduces to

$$
I_{S}^{\left(A_{1}, D_{4}\right)}\left(q^{\frac{1}{2}}\right)=q^{\frac{1}{6} \text { P.E. }}\left(8 \frac{q^{\frac{1}{2}}}{1-q}\right)=\left(I_{S}^{\text {half-hyper }}\right)^{8},
$$

where the righthand side (rhs) is just the index of eight free half-hypermultiplets (i.e., the $T_{2}$ theory [20]) or, equivalently in $2 \mathrm{D}$, the vacuum character of four symplectic bosons.

While the derivation in [15] proved (3) [21] along with various generalizations we will encounter below, we would like to give a physical argument for why this index is so closely related to the index of free fields. One hint comes from the study in [22] (building on [23]) that shows the ( $A_{1}$, $D_{4}$ ) theory plays a role in a particular $S$ duality that is reminiscent of the role played by free hypermultiplets in the $S$ duality of [24]. Moreover, by thinking of (4) as a manifestation of a weak-strong "duality" [25], we, in collaboration with T. Nishinaka, speculated that this connection might be related to modularity [22].

As we will see below, this intuition is morally correct, although the free fields that are more closely related to modularity are actually nonunitary (wrong statistics) rather than the unitary fields appearing on the rhs of (4). A strong indication that this idea is correct comes from noting that (3) satisfies the modular differential equation [26]

$\left.\left(D_{q}^{(2)}-40 \mathbb{E}_{4}\right) I_{S}^{\left(A_{1}, D_{4}\right)}=\left(D_{q}^{(2)}-40 \mathbb{E}_{4}\right) \chi_{0}^{\widehat{s u(3)}}\right)_{-\frac{3}{2}}=0$,

where $D_{q}^{(2)}$ is a modular differential operator, and $\mathbb{E}_{4}$ is an Eisenstein series (we refer the interested reader to [26] for more details). The characters of $\widehat{s o(8)})_{1}$ satisfy the same modular differential equation [27]. Since $\widehat{s o(8)})_{1}$ is unitary and has a representation in terms of eight free Majorana fermions, it is reasonable to imagine that the 4D ancestor of this theory is a nonunitary free theory (recall that, as discussed above, $c_{4 d}=-(1 / 12) c_{2 d}$, so $c_{4 d}<0$ in this case). Clearly, these free fields then reproduce some of the observables in the Schur sector of the $\left(A_{1}, D_{4}\right)$ SCFT.

Modular $S$ transformations and an affine Kac-Moody $(A K M)$ relation.-In order to understand the modular properties of (3), it is useful to rewrite it as follows:

$$
I_{S}^{\left(A_{1}, D_{4}\right)}=2^{-4} \frac{\theta_{2}(\tau)^{4}}{\eta(\tau)^{4}},
$$

where $q=e^{2 \pi i \tau}, \eta(\tau)$ is the Dedekind eta function, and $\theta_{i}(\tau)$ are the Jacobi theta functions (see the Supplemental Material [4]). Under a modular $S$ transformation, we have

$\theta_{2}\left(-\frac{1}{\tau}\right)=\sqrt{-i \tau} \theta_{4}(\tau), \quad \eta\left(-\frac{1}{\tau}\right)=\sqrt{-i \tau} \eta(\tau)$.

In particular, we see that applying a modular $S$ transformation to (6) yields

$$
\mathcal{S}\left(I_{S}^{\left(A_{1}, D_{4}\right)}\right)=2^{-4} \frac{\theta_{4}(\tau)^{4}}{\eta(\tau)^{4}}=2^{-4} q^{-\frac{1}{6} \text { P.E. }}\left(-\frac{8 q^{\frac{1}{2}}}{1-q}\right) .
$$

We immediately recognize the expression on the rhs as also counting [with a $(-1)^{F}$ weighting] the $\left.\widehat{s o(8)}\right)_{1}$ fields generated by acting on the $\widehat{s o(8)}{ }_{1}$ vacuum with the $h=$ $\frac{1}{2}$ Majorana fermions in the $\mathbf{8}_{\mathrm{v}}$ representation, $\psi^{I}$ (where $I=1, \ldots, 8)[1,4]$ (hence, this theory is related to eight decoupled Ising models). These fields have the following singular operator product expansion (OPE):

$$
\psi^{I}(z) \psi^{J}(w) \sim \frac{\delta^{I J}}{z-w} .
$$

At the level of characters, we have the relation

$$
\begin{aligned}
& \mathcal{S}\left(\chi_{0}^{\widehat{s u(3)}}{ }_{-\frac{3}{2}}\right)=-\frac{1}{2}\left(\chi_{0}^{\widehat{\operatorname{su(3)}}}{ }_{-\frac{3}{2}}+\widehat{s u(3)_{-\frac{3}{2}}}+\chi_{-\frac{1}{2}, 1}^{\widehat{s u(3)_{-\frac{3}{2}}}}\right. \\
& \left.-\chi_{-\frac{1}{2}, 3}^{\widehat{s u(3)}}-\frac{-3}{2}\right)=2^{-4}\left(\widehat{\chi_{0}^{s o(8)_{1}}}-\widehat{\chi_{\frac{1}{2}, v}^{s o(8)_{1}}}\right),
\end{aligned}
$$

where, in the second equality, we have used our observation above and, in the first equality, we have used the modular $S$ matrix acting on the characters of the four admissible representations of $\widehat{\operatorname{su(3)}})_{-\frac{3}{2}}$

$$
S_{\widehat{s u(3)}-\frac{3}{2}}=-\frac{1}{2}\left(\begin{array}{cccc}
1 & 1 & 1 & -1 \\
1 & 1 & -1 & 1 \\
1 & -1 & 1 & 1 \\
-1 & 1 & 1 & 1
\end{array}\right) \text {. }
$$

There are four admissible representations of $\widehat{s u(3)})_{-\frac{3}{2}}$ (the vacuum and three $h=-\frac{1}{2}$ representations) and four representations of $\widehat{s o(8)})_{1}$ (the vacuum and three $h=\frac{1}{2}$ representations), but in the latter case, all four corresponding unrefined characters are finite, while in the former case, only two linear combinations of unrefined characters are finite [the vacuum and the linear combination of $h=-\frac{1}{2}$ 
characters, $\chi_{-\frac{1}{2}}^{s u(3)_{-\frac{3}{2}}}$, appearing in (10)]. However, all the unrefined $h=\frac{1}{2}$ characters of $\left.\widehat{s o(8)}\right)_{1}$ are equal (we denote the corresponding character $\left.\chi_{\frac{1}{2}}^{\prime s o(8)_{1}}\right)$, and we find the bijection of finite unrefined characters [28]

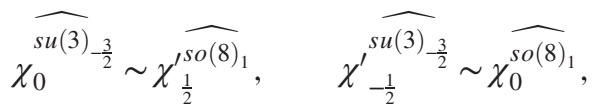

where the relations hold up to overall constants (see [29] for character relations between other pairs of unitary and nonunitary theories).

A 4D interpretation.-We would like to give a 4D interpretation for the unitary $\widehat{s o(8)})_{1}$ theory described in the previous section by using the relation discovered in [3] (although, a priori, it is not clear such an interpretation must exist). As discussed above, this theory should be nonunitary since

$$
c_{\operatorname{so}(8)_{1}}=4 \Rightarrow c_{4 d}=-\frac{1}{3} .
$$

Moreover, from the results in (2) and (8), we see that an obvious candidate for our 4D theory is a collection of 8 half-hypermultiplets with wrong statistics (i.e., a "ghost" $T_{2}$ theory) [30]. Indeed, the $a$ and $c$ anomalies for such a theory are just minus the corresponding anomalies for the $T_{2}$ theory since the wrong statistics leads to an insertion of a factor of -1 in any quantum loop. In particular, we have

$$
\begin{aligned}
& c_{4 d}=-8 \times c_{\text {half-hyper }}=-\frac{1}{3}, \\
& a_{4 d}=-8 \times a_{\text {half-hyper }}=-\frac{1}{6} .
\end{aligned}
$$

Note that $a_{4 d}-c_{4 d}$ is then consistent with the $q \rightarrow 1$ "Cardy" limit of the index [9,26,31,32], and the full (unrefined) Schur index is precisely what we want (see [30]).

To get a map of operators, the correspondence in [3] requires us to take 4D Schur operators, fix them in a plane (with coordinates $z, \bar{z}$ ), and then twist the global $\bar{z}$ conformal transformations with $s u(2)_{R}$. Working in the cohomology of a particular supercharge, $\mathbb{Q}$, then gives a map to 2D chiral algebra operators. This procedure is naturally implemented in the OPE.

For the case at hand, we can build all Schur operators as arbitrary (nonvanishing) products of the $s u(2)_{R}$ highest weight anticommuting scalars of the nonunitary free hypermultiplets, $q^{I}$, and their derivatives. These fields are organized as $q^{i}=Q^{i}$ and $q^{i+4}=\tilde{Q}^{i}$ (with $i=1, \ldots, 4$ ) and live in the following $s u(2)_{R}$ doublets:

$$
\left(\begin{array}{c}
Q^{i} \\
\tilde{Q}^{i \dagger}
\end{array}\right), \quad\left(\begin{array}{c}
\tilde{Q}^{i} \\
-Q^{i \dagger}
\end{array}\right)
$$

We can write a simple Lagrangian for this nonunitary theory (note that the spinors in the hypermultiplets commute while the scalars anticommute)

$\mathcal{L}=-\int d^{4} \theta\left(q^{I \dagger} \Omega_{I J} q^{J}\right)=\int d^{4} \theta\left(\tilde{Q}^{i \dagger} \delta_{i j} Q^{j}-Q^{i \dagger} \delta_{i j} \tilde{Q}^{j}\right)$,

where we have defined

$$
\Omega \equiv\left(\begin{array}{cc}
0_{4 \times 4} & 1_{4 \times 4} \\
-1_{4 \times 4} & 0_{4 \times 4}
\end{array}\right)
$$

Related Lagrangians have been considered in different contexts in $[33,34]$.

The nonvanishing singular OPEs are then (in an appropriate normalization to eliminate a common overall constant factor)

$$
\tilde{Q}^{i \dagger}(x) Q^{j}(0) \sim \frac{\delta^{i j}}{x^{2}}, \quad Q^{i \dagger}(x) \tilde{Q}^{j}(0) \sim-\frac{\delta^{i j}}{x^{2}}
$$

According to the discussion in [3], we should twist the hypermultiplets with vectors $u_{i}=(1, \bar{z})$ having $s u(2)_{R}$ indices $i=1,2$. In particular, we have twisted fields

$$
\begin{aligned}
& Q^{\prime i}(z, \bar{z})=Q^{i}(z, \bar{z})+\bar{z} \tilde{Q}^{i \dagger}(z, \bar{z}), \\
& \tilde{Q}^{\prime i}(z, \bar{z})=\tilde{Q}^{i}(z, \bar{z})-\bar{z} Q^{i \dagger}(z, \bar{z}),
\end{aligned}
$$

with the following singular OPEs:

$$
\begin{aligned}
& Q^{\prime i}(z, \bar{z}) Q^{\prime j}(0,0) \sim \frac{\delta^{i j}}{z} \\
& \tilde{Q}^{\prime i}(z, \bar{z}) \tilde{Q}^{\prime j}(0,0) \sim \frac{\delta^{i j}}{z}
\end{aligned}
$$

Passing to $\mathbb{Q}$ cohomology gives the same OPEs as above (the identity operator is $\mathbb{Q}$ closed but, clearly, cannot be $\mathbb{Q}$ exact). In particular, we reproduce the free Majorana OPEs of (9).

The theory also has conserved currents sitting as leveltwo descendants in multiplets with Schur operators of the form

$\mu^{i j}=i Q^{i} Q^{j}, \quad \tilde{\mu}^{i j}=i \tilde{Q}^{i} \tilde{Q}^{j}, \quad \mu^{i j}=i Q^{i} \tilde{Q}^{j}$,

where $i, j=1, \ldots, 4$. More covariantly, we can define these operators to form part of a 28-dimensional adjoint representation with $\mu^{I J}=i q^{I} q^{J}$ and $I, J=1, \ldots, 8$ (this operator is antisymmetric in $I$ and $J$ ). The charges arising from real currents sitting as descendants of linear combinations of the 
above satisfy an $s o^{*}(8) \simeq \operatorname{so}(6,2)$ Lie algebra, which is a real form of $s o(8, \mathbb{C})$. On the other hand, the operators in (21) are related to currents that are not real. However, these currents give rise to charges that act in accordance with the reality condition in two dimensions

$$
\begin{aligned}
& \mu^{i j}: \delta Q^{i} \sim-Q^{j}, \delta Q^{j} \sim Q^{i}, \delta \tilde{Q}^{i \dagger} \sim-\tilde{Q}^{j \dagger}, \delta \tilde{Q}^{j \dagger} \sim \tilde{Q}^{i \dagger}, \\
& \tilde{\mu}^{i j}: \delta \tilde{Q}^{i} \sim \tilde{Q}^{j}, \delta \tilde{Q}^{j} \sim-\tilde{Q}^{i}, \delta Q^{i \dagger} \sim Q^{j \dagger}, \delta Q^{j \dagger} \sim-Q^{i \dagger}, \\
& \mu^{\prime i j}: \delta \tilde{Q}^{j} \sim Q^{i}, \delta Q^{i} \sim-\tilde{Q}^{j}, \delta \tilde{Q}^{i \dagger} \sim Q^{j \dagger}, \delta Q^{j \dagger} \sim-\tilde{Q}^{i \dagger} .
\end{aligned}
$$

Relabeling the moment maps with an adjoint index of so(8), we obtain the following twisted OPE:

$$
\mu^{A}(z, \bar{z}) \mu^{B}(0) \sim \frac{\delta^{A B}}{z^{2}}+i f_{C}^{A B} \frac{\mu^{C}(0,0)}{z}+\{\mathbb{Q}, \ldots\},
$$

where $f_{C}^{A B}$ are the structure constants of so(8). Dropping the $\mathbb{Q}$-exact terms then leads to the standard $\widehat{s o(8)})_{1}$ currentcurrent OPE. As a result, we see that a generalization of the procedure of [3] applied to our nonunitary 4D theory yields the desired unitary theory in 2D.

Infinitely many generalizations.-One can imagine generalizing our discussion above in many directions. Here, we choose the simplest direction: the $\left(A_{1}, D_{4}\right)$ theory is part of an infinite family of SCFTs called the $D_{2}[S U(2 N+1)]$ theories $[35,36]$ [where $\left.D_{2}[S U(3)] \equiv\left(A_{1}, D_{4}\right)\right]$. The corresponding chiral algebras were found in [14] and were argued to be $s u(2 \widehat{N+1})_{-(2 N+1 / 2)}$. The generalization of (3) is

$I_{S}^{D_{2}[S U(2 N+1)]}(q)=q^{N(N+1) / 6}$ P.E. $\left(4 N(N+1) \frac{q}{1-q^{2}}\right)$,

and one finds that, upon taking $q \rightarrow q^{\frac{1}{2}}$, the index (23) reduces to the index of $4 N(N+1)$ free half-hypermultiplets.

For $N>1$, the modular properties of the theory are somewhat different. For example, the modular differential equation in (5) for the $N=1$ case becomes third order for all $N>1$. However, we can proceed as before and write

$$
I_{S}^{D_{2}[S U(2 N+1)]}=2^{-2 N(N+1)} \frac{\theta_{2}(\tau)^{2 N(N+1)}}{\eta(\tau)^{2 N(N+1)}} .
$$

Then, performing a modular $S$ transformation yields

$$
\begin{aligned}
\mathcal{S} & \left(I_{S}^{D_{2}[S U(2 N+1)]}\right) \\
& =2^{-2 N(N+1)} \frac{\theta_{4}(\tau)^{2 N(N+1)}}{\eta(\tau)^{2 N(N+1)}} \\
& =2^{-2 N(N+1)} q^{-[N(N+1) / 12]} \text { P.E. }\left(-\frac{4 N(N+1) q^{\frac{1}{2}}}{1-q}\right) .
\end{aligned}
$$

This result generalizes the $N=1$ result discussed above, since we recognize (25) as also counting [with a $(-1)^{F}$ weighting] the $s o[4 \widehat{N(N}+1)]_{1}$ fields generated by acting on the $s o[4 \widehat{N(N}+1)]_{1}$ vacuum with the $h=\frac{1}{2}$ Majorana fermion in the vector representation, $\psi^{I}$ [its singular self-OPE is the obvious generalization of (9) with $I=1, \ldots, 4 N(N+1)]$.

The $\operatorname{so}[4 \widehat{(N}+1)]_{1}$ algebra has four representations for all $N$ : the vacuum, the $h=1 / 2$ representation discussed above, and two $h=N(N+1) / 4$ representations. The latter two representations have identical unrefined characters which we denote as $\chi_{N(N+1) / 4}^{\prime s o(\widehat{4 N[N}+1)]_{1}}$ (for $N=1$, the last three unrefined characters are identical). On the other hand, the $s u(\widehat{2 N+1})_{-(2 N+1 / 2)}$ algebra has three finite (linear combinations of) unrefined characters that transform into each other under modular transformations: one starting with $h=$ 0 (the vacuum), one starting with $h=(2-N(N+1) / 4)$, and one starting with $h=-[N(N+1) / 4]$. It is straightforward to check that

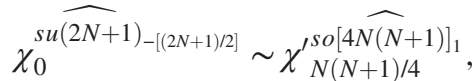

$$
\begin{aligned}
& \chi^{\prime s u(2 N+1)_{-[(2 N+1) / 2]}} \sim \chi_{\frac{1}{2}}^{s o[4 N(N+N+1)]_{1}}, \\
& \chi_{-[N(N+1) / 4]}^{\text {su }} \widehat{(2 N+1)_{-[(2 N+1) / 2]}} \sim \chi_{0}^{s o\left[4 N \widehat{(N+1)]_{1}}\right.} .
\end{aligned}
$$

These results are simple consequences of the fact that our two chiral algebras satisfy the same modular differential equation for all $N$.

The 4D generalization of the $N=1$ case is straightforward. For example, we have that

$c_{s o[4 N(N+1)]_{1}}=2 N(N+1) \Rightarrow c_{4 d}=-\frac{N(N+1)}{6}$.

This anomaly is precisely what we expect for $4 N(N+1)$ half-hypermultiplets with wrong statistics [i.e., $N(N+1) / 2$ "ghost" $T_{2}$ theories]. Similarly, $a_{4 d}$ and the superconformal index are compatible with this interpretation. In particular, our 4D Lagrangian is just the obvious generalization of (16)

$$
\mathcal{L}=-\int d^{4} \theta\left(q^{I \dagger} \Omega_{I J} q^{J}\right)=\int d^{4} \theta\left(\tilde{Q}^{i \dagger} \delta_{i j} Q^{j}-Q^{i \dagger} \delta_{i j} \tilde{Q}^{j}\right),
$$

where now $I=1, \ldots, 4 N(N+1)$. Note that the real flavor currents in $4 \mathrm{D}$ generate an $s o^{*}[4 N(N+1)]$ algebra, but the $N(2 N-1)$ Schur operators that are the generalizations of (21) give rise to the $s o[4 \widehat{N(N}+1)]_{1}$ AKM algebra in 2D [37]. 
Discussion and conclusions.-We have seen that the simple nonunitary 4D Lagrangian (28) allows us (through manipulations in two dimensions) to exactly compute the unrefined Schur indices for the $D_{2}[S U(2 N+1)]$ SCFTs. Clearly, we are also able to compute other (linear combinations of) characters of the associated chiral algebras via the, to our knowledge, novel mathematical identities in (26). Based on known relations between chiral algebras in 2D and 3D QFT, it is reasonable to expect that aspects of the physics of the nonvacuum modules of the chiral algebras are captured by (worldvolumes of) 4D objects that have nontrivial braiding statistics as in [38]. Indeed, there is considerable evidence that this intuition holds $[39,40]$, and we hope to return to a detailed discussion of surface and line defects in our setup soon. In particular, the Lagrangian in (28) seems to compute the Schur indices of the $D_{2}[S U(2 N+1)]$ SCFTs in the presence of certain surface defects [41], while we, presumably, need to introduce defects in our nonunitary theory in order to compute-directly in 4D-the other Schur indices of the $D_{2}[S U(2 N+1)]$ theories.

As another future direction, we may hope to find information about new observables that are closely related to the chiral algebra as in [42-45]. Moreover, since we have a Lagrangian description of certain Schur observables, it is tempting to see what (if anything) the corresponding correlation functions or OPE coefficients compute in the original strongly interacting theory. Even more simply, it would be interesting to understand whether it is possible to map flavor symmetries between our two sets of theories.

Moreover, we expect that our procedure of starting with a unitary $4 \mathrm{D}$ theory, mapping to 2D using [3], conjugating or permuting the characters, reinterpreting the characters as objects in a unitary 2D theory, and then lifting to a nonunitary theory in 4D will generalize (with certain modifications) to many (and perhaps all) $\mathcal{N}=2$ theories. While we know that the nonunitary 4D theories will not always have a completely free description in terms of hypermultiplets, we expect gauge fields and perhaps the constructions in [46] to play a role (possibly when the original 4D theory has a conformal manifold [47-51]). Indeed, we expect nonunitary Lagrangians to be a more diverse and flexible group of objects than their unitary counterparts, and so, we expect them to describe "more" theories.

Still, we should point out that our nonunitary 4D theories described above have an avatar of 2D unitarity: a modified notion of reflection positivity exists in our theories. Related 2D constructions have played a role in recent work on nonunitary extensions of Zamolodchikov's $c$ theorem [52]. Such structures, involving "hidden" unitarity, may also shed more light on the question of which nonunitary theories in 2D are able to encode the unitary 4D physics in the original construction of [3]. We hope to return to this question soon.
It would also be interesting to understand any relation between our construction and the Lagrangians appearing in [53-60]. While our Lagrangians govern only a particular sector of the theories we study (and perhaps only a particular set of observables in such a sector), they are considerably simpler than the "full" Lagrangians in these latter works [61]. Also, our approach is different: we sacrifice unitarity instead of the $\mathcal{N}=2$ superconformal algebra. More generally, it would be interesting to find connections between our discussion and other effective Lagrangian descriptions of sectors of QFTs (e.g., as in [62]).

Finally, we hope to understand whether our work is related in any way to supersymmetric localization (see [40] for some interesting work in this direction from a chiral algebra perspective), to understand whether our work is related to the free fermion description of the Schur index for quiver gauge theories [63], to see how our procedure might work in $6 \mathrm{D}$, to understand whether the theories we have studied here contain some sectors that play a role in the dS/CFT correspondence, and to understand the role our Lagrangians might play in the physics of 3D SCFTs as in $[58,59,64]$.

We are grateful to T. Nishinaka for many interesting discussions-including drawing our attention to the formula in [14] when one of us (M. B.) visited Kyoto last year - and also, for many collaborations on related topics. We also thank S. Giacomelli and M. Roberts for correspondence and discussions. M. B.'s research is partially supported by the Royal Society under the "New Constraints and Phenomena in Quantum Field Theory Grant." Z. L. is supported by Queen Mary University of London.

[1] P. Di Francesco, P. Mathieu, and D. Senechal, Conformal Field Theory (Springer-Verlag, New York, 1997).

[2] We will not make this notion of smoothness precise here, except to say that, at the very least, there should not be any accidental symmetries along the resulting renormalization group flow that obscure the observable one would like to compute.

[3] C. Beem, M. Lemos, P. Liendo, W. Peelaers, L. Rastelli, and B. C. van Rees, Infinite chiral symmetry in four dimensions, Commun. Math. Phys. 336, 1359 (2015).

[4] See Supplemental Material at http://link.aps.org/ supplemental/10.1103/PhysRevLett.120.081601 for a brief description of the characters of $s o(2 n)_{1}$.

[5] A. Gadde, L. Rastelli, S. S. Razamat, and W. Yan, Gauge theories and macdonald polynomials, Commun. Math. Phys. 319, 147 (2013).

[6] This theory was originally discovered in [7], but we use the naming conventions of [8].

[7] P. C. Argyres, M. R. Plesser, N. Seiberg, and E. Witten, New $N=2$ superconformal field theories in four dimensions, Nucl. Phys. B461, 71 (1996). 
[8] S. Cecotti, A. Neitzke, and C. Vafa, R-twisting and $4 d / 2 d$ correspondences, arXiv:1006.3435.

[9] M. Buican and T. Nishinaka, On the superconformal index of Argyres-Douglas theories, J. Phys. A 49, 015401 (2016).

[10] M. Buican and T. Nishinaka, Argyres-Douglas theories, $\mathrm{S}^{1}$ reductions, and topological symmetries, J. Phys. A 49, 045401 (2016).

[11] C. Cordova and S. H. Shao, Schur Indices, BPS particles, and Argyres-Douglas theories, J. High Energy Phys. 01 (2016) 040.

[12] M. Buican and T. Nishinaka, Argyres-Douglas theories, the Macdonald index, and an RG inequality, J. High Energy Phys. 02 (2016) 159.

[13] L. Rastelli, Harvard University, 2014 (unpublished).

[14] D. Xie, W. Yan, and S. T. Yau, Chiral algebra of ArgyresDouglas theory from M5 brane, arXiv:1604.02155.

[15] V. G. Kac and M. Wakimoto, A remark on boundary level admissible representations, Comptes Rendus Mathematique 355, 128 (2017).

[16] T. Creutzig, W-algebras for Argyres-Douglas theories, arXiv: 1701.05926.

[17] The authors of [18] also argued in favor of (3) (and a generalization we will encounter below) using results in $[9,19]$.

[18] J. Song, D. Xie, and W. Yan, Vertex operator algebras of Argyres-Douglas theories from M5-branes, J. High Energy Phys. 12 (2017) 123.

[19] J. Song, Superconformal indices of generalized ArgyresDouglas theories from 2d TQFT, J. High Energy Phys. 02 (2016) 045.

[20] D. Gaiotto, $N=2$ dualities, J. High Energy Phys. 08 (2012) 034.

[21] More precisely, the authors of [15] proved that the vacuum character of $\widehat{s u(3)_{-\frac{3}{2}}}$ is given by the rhs of (3).

[22] M. Buican, Z. Laczko, and T. Nishinaka, $\mathcal{N}=2$ S-duality revisited, J. High Energy Phys. 09 (2017) 087.

[23] M. Buican, S. Giacomelli, T. Nishinaka, and C. Papageorgakis, Argyres-Douglas theories and S-duality, J. High Energy Phys. 02 (2015) 185.

[24] P. C. Argyres and N. Seiberg, S-duality in $N=2$ supersymmetric gauge theories, J. High Energy Phys. 12 (2007) 088.

[25] The relation in (4) is not a duality in the truest sense of the word since it is a relation between the Schur sectors of two different theories.

[26] C. Beem and L. Rastelli, Vertex operator algebras, Higgs branches, and modular differential equations, arXiv: 1707.07679.

[27] S. D. Mathur, S. Mukhi, and A. Sen, On the classification of rational conformal field theories, Phys. Lett. B 213, 303 (1988).

[28] Ultimately, the fact that there are only two characters transforming amongst each other under modular transformations in the case of our two AKM algebras is a consequence of the Jacobi quartic identity $\theta_{3}(\tau)^{4}=$ $\theta_{2}(\tau)^{4}+\theta_{4}(\tau)^{4}$

[29] S. Mukhi and S. Panda, Fractional level current algebras and the classification of characters, Nucl. Phys. B338, 263 (1990).
[30] To get just the $\widehat{s o(8)}$, vacuum module, we should restrict to composite Schur operators built from an even number of hypermultiplet scalars.

[31] L. Di Pietro and Z. Komargodski, Cardy formulae for SUSY theories in $d=4$ and $d=6$, J. High Energy Phys. 12 (2014) 031.

[32] A. Arabi Ardehali, High-temperature asymptotics of supersymmetric partition functions, J. High Energy Phys. 07 (2016) 025.

[33] D. Anninos, T. Hartman, and A. Strominger, Higher spin realization of the DS/CFT correspondence, Classical Quantum Gravity 34, 015009 (2017).

[34] T. Hertog, G. Tartaglino-Mazzucchelli, T. Van Riet, and G. Venken, Supersymmetric dS/CFT, arXiv:1709.06024.

[35] S. Cecotti and M. Del Zotto, Infinitely many $N=2$ SCFT with ADE flavor symmetry, J. High Energy Phys. 01 (2013) 191.

[36] S. Cecotti, M. Del Zotto, and S. Giacomelli, More on the $N=2$ superconformal systems of type $D_{p}(G)$, J. High Energy Phys. 04 (2013) 153.

[37] To get just the $\operatorname{so}[4 \widehat{N(N}+1)]_{1}$ vacuum module, we should restrict to composite Schur operators built from an even number of $q^{I}$.

[38] C. Wang and M. Levin, Braiding Statistics of Loop Excitations in Three Dimensions, Phys. Rev. Lett. 113, 080403 (2014).

[39] C. Cordova, D. Gaiotto, and S. H. Shao, Surface defects and chiral algebras, J. High Energy Phys. 05 (2017) 140.

[40] Y. Pan and W. Peelaers, Chiral algebras, localization and surface defects, arXiv:1710.0430.

[41] Or, more precisely, it computes linear combinations of the usual Schur indices and the Schur indices in the presence of certain surface defects.

[42] J. Song, Macdonald index and chiral algebra, J. High Energy Phys. 08 (2017) 044.

[43] L. Fredrickson, D. Pei, W. Yan, and K. Ye, Argyres-Douglas theories, chiral algebras and wild hitchin characters, arXiv:1701.08782.

[44] M. Fluder and J. Song, Four-dimensional lens space index from two-dimensional chiral algebra, arXiv:1710.06029.

[45] Y. Imamura, Orbifold Schur index and IR formula, arXiv: 1710.08853 .

[46] R. Dijkgraaf, B. Heidenreich, P. Jefferson, and C. Vafa, Negative branes, supergroups and the signature of spacetime, arXiv:1603.05665.

[47] M. Buican and T. Nishinaka, Conformal manifolds in four dimensions and chiral algebras, J. Phys. A 49, 465401 (2016).

[48] M. Buican and T. Nishinaka, On irregular singularity wave functions and superconformal indices, J. High Energy Phys. 09 (2017) 066.

[49] D. Xie and S. T. Yau, Argyres-Douglas matter and $N=2$ dualities, arXiv:1701.01123.

[50] D. Xie and K. Ye, Argyres-Douglas matter and S-duality: Part II, arXiv:1711.06684.

[51] J. Choi and T. Nishinaka, On the chiral algebra of ArgyresDouglas theories and S-duality, arXiv:1711.07941.

[52] O. A. Castro-Alvaredo, B. Doyon, and F. Ravanini, Irreversibility of the renormalization group flow in non-unitary quantum field theory, J. Phys. A 50, 424002 (2017). 
[53] A. Gadde, S. S. Razamat, and B. Willett, "Lagrangian" for a Non-Lagrangian Field Theory with $\mathcal{N}=2$ Supersymmetry, Phys. Rev. Lett. 115, 171604 (2015).

[54] K. Maruyoshi and J. Song, Enhancement of Supersymmetry via Renormalization Group Flow and the Superconformal Index, Phys. Rev. Lett. 118, 151602 (2017).

[55] K. Maruyoshi and J. Song, $\mathcal{N}=1$ deformations and RG flows of $\mathcal{N}=2$ SCFTs, J. High Energy Phys. 02 (2017) 075.

[56] P. Agarwal, K. Maruyoshi, and J. Song, $\mathcal{N}=1$ Deformations and RG flows of $\mathcal{N}=2$ SCFTs, part II: non-principal deformations, J. High Energy Phys. 12 (2016) 103; Addendum: $\mathcal{N}=1$ Deformations and RG flows of $\mathcal{N}=2$ SCFTs, part II: non-principal deformations, J. High Energy Phys. 04 (2017) 113.

[57] P. Agarwal, A. Sciarappa, and J. Song, $\mathcal{N}=1$ Lagrangians for generalized Argyres-Douglas theories, J. High Energy Phys. 10 (2017) 211.
[58] S. Benvenuti and S. Giacomelli, Abelianization and sequential confinement in $2+1$ dimensions, J. High Energy Phys. 10 (2017) 173.

[59] S. Benvenuti and S. Giacomelli, Lagrangians for generalized Argyres-Douglas theories, J. High Energy Phys. 10 (2017) 106.

[60] S. Giacomelli, RG flows with supersymmetry enhancement and geometric engineering, arXiv:1710.06469.

[61] Although, at present, there are no known "full" Lagrangians for the $D_{2}[S U(2 N+1)]$ theories with $N>1$.

[62] S. Hellerman and S. Maeda, On the large $R$-charge expansion in $\mathcal{N}=2$ superconformal field theories, J. High Energy Phys. 12 (2017) 135.

[63] J. Bourdier, N. Drukker, and J. Felix, The $\mathcal{N}=2$ Schur index from free fermions, J. High Energy Phys. 01 (2016) 167.

[64] N. Aghaei, A. Amariti, and Y. Sekiguchi, Notes on integral identities for 3d supersymmetric dualities, arXiv:1709.08653. 